\title{
The Prospects for Yb- and Nd-Doped Tungstate Microchip Lasers
}

\author{
V.G. Savitski ${ }^{1}$, R.B. Birch ${ }^{1}$, E. Fraczek ${ }^{1}$, A.J. Kemp ${ }^{1}$, P.A. Loiko ${ }^{2}$, K.V. Yumashev ${ }^{2}$, N.V. Kuleshov ${ }^{2}$, A.A. Pavlyuk ${ }^{3}$ \\ 1. Institute of Photonics, University of Strathclyde, SUPA, 106 Rottenrow, Glasgow, G4 ONW, UK \\ 2. Center for Optical Materials and Technologies, Belarusian National Technical University, 220013 Belarus, Minsk, 65/17 Nezavisimosti Ave. \\ 3. A.V. Nikolaev Institute for Inorganic Chemistry, Siberian Branch of RAS, 630090 Russia, Novosibirsk, 3 Lavrentyev Ave.
}

Potassium gadolinium and yttrium tungstates doped with trivalent $\mathrm{Nd}, \mathrm{Yb}$ or $\mathrm{Tm}$ ions are widely used crystals for efficient diode-pumped lasers. They are usually oriented along the $b$ crystallographic axis, which is also the $N_{\mathrm{p}}$ axis of optical indicatrix. The $N_{\mathrm{p}}$-cut is characterized by a strong thermal lens with opposing signs along $N_{\mathrm{m}}$ and $N_{\mathrm{g}}$ [1]. This is unsuited to microchip lasers which require a positive thermal lens for a stable cavity. Therefore, a detailed investigation of thermo-optic effects and microchip laser potential of other crystallographic orientations in tungstate crystals is of interest.

$N_{\mathrm{g}}$-cut tungstate crystals provide a positive and near-spherical thermal lens [1]. This enabled the demonstration of a $\mathrm{N}_{\mathrm{g}}$ cut Tm:KY $\left(\mathrm{WO}_{4}\right)_{2}(\mathrm{KYW})$ laser in a plane-plane cavity at $1.94 \mu \mathrm{m}$ [2]. In this study, we present for the first time, to the best of our knowledge, the results of $\mathrm{CW}$ laser performance of $N_{\mathrm{g}}$-cut Nd: $\mathrm{KGd}\left(\mathrm{WO}_{4}\right)_{2}(\mathrm{KGW})$ and $\mathrm{Yb}: \mathrm{KYW}$ crystals in a quasi-microchip plane-plane cavity. Proof-of-concept quasi-microchip lasers were demonstrated using 3 at. \% $\mathrm{Nd}: \mathrm{KGd}\left(\mathrm{WO}_{4}\right)_{2}(\mathrm{KGW})$ and 10 at. \% Yb:KYW crystals cut for light propagation along the $N_{\mathrm{g}}$ axis. Both crystals were uncoated and had a thickness of $250 \mu \mathrm{m}$. The samples were capillary-bonded between the two synthetic diamonds (sandwich configuration, see Fig. 1 (a)) for better thermal management. Diamond heat-spreader \#1 was antireflection (AR) coated at 808 and $1064 \mathrm{~nm}$ in the case of Nd:KGW crystal and was uncoated in the case of the Yb:KYW crystal. Diamond heat-spreader \#2 was coated for high reflection (HR) within $808-1064 \mathrm{~nm}$. The composite samples were mounted in brass mounts chilled to $10^{\circ} \mathrm{C}$ via water cooling. The total cavity length was $\sim 2 \mathrm{~mm}$ in both cases. $10 \mathrm{~W}$ fibre-coupled $(200 \mu \mathrm{m}$ core diameter) diode lasers at 808 (Nd:KGW) or $980 \mathrm{~nm}$ (Yb:KYW) were used as pump sources. The pump spot radii in the Nd:KGW and $\mathrm{Yb}: \mathrm{KYW}$ crystals were 150 and $125 \mu \mathrm{m}$, respectively. The best slope efficiency and output power of the Nd:KGW laser were $33 \%$ and $906 \mathrm{~mW}$ respectively (fig. 1(b)), both for an output coupling of $2.4 \%$ and limited by debonding of the sandwich structure. For Yb:KYW, the best slope efficiency and output power were $41 \%$ and $795 \mathrm{~mW}$ respectively, for a $2 \%$ output coupling and limited by the available pump power. Improvements in the bonding and optimisation of the coatings should significantly improve performance of these lasers.

Initial theoretical estimates of thermal lens sensitivity factors $M=d D / d P_{\text {abs }}$ ( $D$ is the thermal lens strength and $P_{\text {abs }}$ is the absorbed pump power [2]) for $N_{\mathrm{g}}$-cut $\mathrm{Nd}: \mathrm{KGW}$ and Yb:KYW, taking into account the diamond heat-spreaders bonded to the crystals, are presented in Fig.1 (c, d). For the Nd:KGW laser, the thermal lens at an absorbed pump power of 4W was estimated from Fig. 1 (c) to be $\sim 50 \mathrm{~mm}$, giving a TEM $\mathrm{T}_{00}$ mode radius of $\sim 60 \mu \mathrm{m}$, which is smaller than the pump spot radius of $150 \mu \mathrm{m}$ and therefore further optimisation of the laser is required. In the case of $\mathrm{Yb}: \mathrm{KYW}$ laser and for laser emission polarised along the $N_{\mathrm{m}}$ axis, the thermal lens at an absorbed pump power of $4 \mathrm{~W}$ is $\sim 570 \mathrm{~mm}$ (from Fig. 1 (d)), giving a $\mathrm{TEM}_{00}$ mode radius of $\sim 110 \mu \mathrm{m}$. This is close to the pump spot radius of $125 \mu \mathrm{m}$ suggesting the pump radius may be close to optimised for this absorbed pump power. Indeed, with the smaller pump spot radius of $100 \mu \mathrm{m}$ the output power of the laser drops and the same effect, although not as marked, was observed when the pump spot radius was increased to $160 \mu \mathrm{m}$.

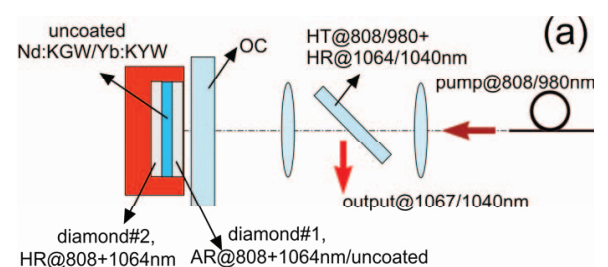

HR@808+1064nm AR@808+1064nm/uncoated
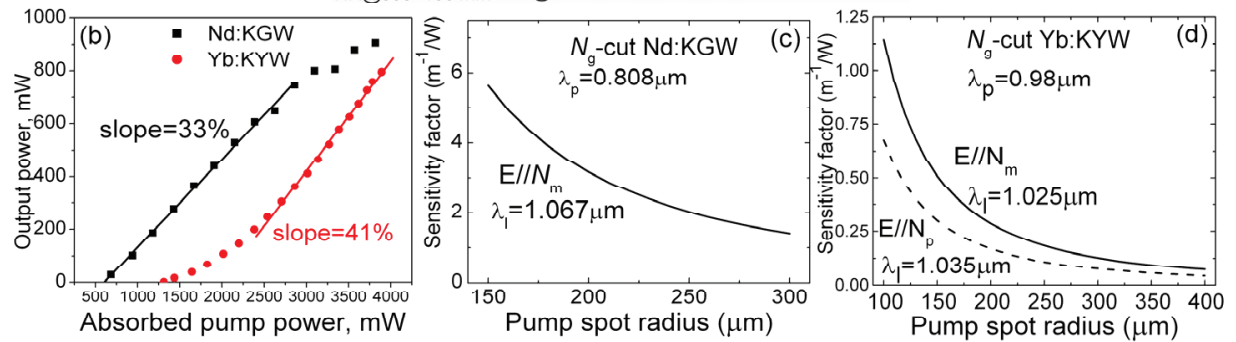

Fig. 1 (a) Quasi-microchip laser setup : OC - output coupler; (b) output powers of Nd :KGW and Yb :KYW quasimicrochip lasers as function of absorbed pump power; (c) calculated sensitivity factor of thermal lens in $N_{\mathrm{g}}$-cut Nd:KGW crystals for light polarisation along $N_{\mathrm{m}}$ axis; (d) calculated sensitivity factor of thermal lens in $N_{\mathrm{g}}$-cut Yb:KYW crystals for light polarisation along $N_{\mathrm{m}}$ and $N_{\mathrm{p}}$ axes.

\section{References}

[1] P.A. Loiko, K.V. Yumashev, N.V. Kuleshov, V.G. Savitski, S. Calvez, D. Burns, A.A. Pavlyuk, "Thermal lens study in diode pumped $N_{g}$ - and $N_{p}$-cut $\mathrm{Nd}: \mathrm{KGd}\left(\mathrm{WO}_{4}\right)_{2}$ laser crystals," Opt. Express 19, 23536 (2009).

[2] M. S. Gaponenko, P. A. Loiko, N. V. Gusakova, K. V. Yumashev, N. V. Kuleshov, and A. A. Pavlyuk, "Thermal lensing and microchip laser performance of $N_{\mathrm{g}}$-cut $\mathrm{Tm}^{3+}: \mathrm{KY}\left(\mathrm{WO}_{4}\right)_{2}$ crystal," Applied Physics B, 108, 603 (2012). 